\title{
Terapia nutricional domiciliar: uma revisão
}

\author{
Home nutrition therapy: a review \\ Terapia nutricional em el hogar: una revisión
}

Recebido: 25/01/2022 | Revisado: 29/01/2022 | Aceito: 17/02/2022 | Publicado: 25/02/2022

\author{
Marciele Alves Bolognese \\ ORCID: https://orcid.org/0000-0003-3417-9566 \\ Universidade Estadual de Maringá, Brasil \\ E-mail: mafb-2006@hotmail.com \\ Patrícia Magalhães de Souza \\ ORCID: https://orcid.org/0000-0001-5916-0744 \\ Universidade Estadual de Maringá, Brasil \\ E-mail: patricia.magalhaes11@ hotmail.com \\ Vanessa Javera Castanheira Néia \\ ORCID: https://orcid.org/0000-0003-2573-3457 \\ Universidade Estadual de Maringá, Brasil \\ E-mail: nutrivanjavera@ hotmail.com \\ Oscar Oliveira Santos \\ ORCID: https://orcid.org/0000-0002-9631-8480 \\ Universidade Estadual de Maringá, Brasil \\ E-mail: oliveirasantos.oscardeoliveira@gmail.com \\ Jesuí Vergílio Visentainer \\ ORCID: https://orcid.org/0000-0003-3412-897X \\ Universidade Estadual de Maringá, Brasil \\ E-mail: jesuivv@gmail.com
}

\begin{abstract}
Resumo
A terapia nutricional domiciliar (TND), pode ser definida como um método assistencial eficaz, utilizado para a recuperação do aporte nutricional de indivíduos não saudáveis, e também, como subsídio nutricional e clínico para tais pacientes, que se encontram em suas próprias casas ou em instituições de repouso. Para o início da TND, é indispensável a atuação de um nutricionista qualificado que entenda a necessidade do paciente. Logo, o objetivo da presente revisão foi discutir as evidências científicas referentes a terapia nutricional domiciliar. A pesquisa foi realizada envolvendo publicações sobre nutrição oral/enteral/parenteral, terapia nutricional domiciliar, assistência dos órgãos de saúde, e redução dos leitos hospitalares nas plataformas, PubMed (US National Library of Medicine), Scientific Electronic Library Online Brasil (SciELO), Web of Science e Portal Periodicos Capes dos últimos 15 anos. Os resultados evidenciam que a TND diminui as complicações e a mortalidade, além de proporcionar ao paciente maior conforto e a oportunidade de se recuperar em seu ambiente familiar.
\end{abstract}

Palavras-chave: Nutrição domiciliar; Dieta oral; Dieta enteral; Dieta parenteral; Método assistencial; Nutrição; Ocupação hospitalar.

\begin{abstract}
Home nutritional therapy (HNT) can be defined as an effective care method, used for the recovery of the nutritional support of unhealthy individuals, and also as a nutritional and clinical subsidy for such patients, who are in their own homes or in hospitals. rest institutions. For the beginning of HNT, the performance of a qualified nutritionist who understands the patient's needs is essential. Therefore, the objective of the present review was to discuss the scientific evidence regarding home nutritional therapy. The research was carried out involving publications on oral/enteral/parenteral nutrition, home nutritional therapy, assistance from health agencies, and reduction of hospital beds on the platforms, PubMed (US National Library of Medicine), Scientific Electronic Library Online Brazil (SciELO), Web of Science and Portal Periodicos Capes of the last 15 years. The results show that HNT reduces complications and mortality, in addition to providing the patient with greater comfort and the opportunity to recover in their family environment.
\end{abstract}

Keywords: Home nutrition; Oral diet; Enteral diet; Parenteral diet; Assistance method; Nutrition; Hospital occupation.

\section{Resumen}

La Terapia Nutricional Domiciliaria (TND) puede definirse como un método de atención eficaz, utilizado para la recuperación del soporte nutricional de individuos enfermos, y también como un subsidio nutricional y clínico para dichos pacientes, que se encuentran en sus propios domicilios o en hospitales. instituciones Para el inicio de TND, es 
fundamental la actuación de un nutricionista calificado que comprenda las necesidades del paciente. Por lo tanto, el objetivo de la presente revisión fue discutir la evidencia científica sobre la terapia nutricional domiciliaria. La investigación se realizó involucrando publicaciones sobre nutrición oral/enteral/parenteral, terapia nutricional domiciliaria, asistencia de agencias de salud y reducción de camas hospitalarias en las plataformas, PubMed (Biblioteca Nacional de Medicina de EE. UU.), Scientific Electronic Library Online Brasil (SciELO), Web of Science y Portal Periodicos Capes de los últimos 15 años. Los resultados muestran que el TND reduce las complicaciones y la mortalidad, además de brindar al paciente mayor comodidad y la oportunidad de recuperarse en su entorno familiar.

Palabras clave: Nutrición en el hogar; Dieta oral; Dieta enteral; Dieta parenteral; Método de asistencia; Nutrición; Ocupación hospitalaria.

\section{Introdução}

Para o indivíduo manter o bom funcionamento de seu organismo, a alimentação se torna um fator primordial e extremamente importante, especialmente para aqueles acometidos por algum tipo de enfermidade. Nesses casos, um plano de cuidado nutricional diferenciado é necessário, podendo esse, ser realizado no ambiente domiciliar através da Terapia Nutricional Domiciliar (TND) (Cutchma et al., 2016).

A TND, é definida como um método assistencial eficaz, utilizado para a recuperação do aporte nutricional de indivíduos não saudáveis, e também, como auxílio nutricional e clínico para pacientes, que se encontram em suas próprias casas ou em instituições de repouso. A mesma pode ser instituída de forma oral, enteral ou parenteral (Ribas et al., 2016).

A Terapia Nutricional Domiciliar Oral (TNDO), é indicada quando a alimentação convencional não é suficiente para atender às necessidades nutricionais. Nesse caso, é recomendado o uso de suplementos ou alimentos especialmente formulados e processados, nos quais são introduzidas modificações na composição em macro e micronutrientes, visando atender as necessidades de pessoas com condições metabólicas e fisiológicas específicas. Esses suplementos podem ser usados como única fonte de alimentação ou como complemento da alimentação convencional (Coppini et al., 2009). Já a Terapia Nutricional Domiciliar Enteral (TNDE), se caracteriza por ser um conjunto de procedimentos terapêuticos que corroboram para a recuperação nutricional dos pacientes que estão impossibilitados de alcançar suas necessidades via alimentação oral, garantindo a funcionalidade íntegra do organismo (Szlagatys-Sidorkiewicz et al., 2012). E por fim, a Terapia Nutricional Domiciliar Parenteral (TNDP) é amplamente indicada para aqueles pacientes que estão incapacitados de atingir suas necessidades nutricionais via oral e enteral, ou seja, casos em que o sistema digestório está comprometido, e por consequência a absorção dos nutrientes é incompleta (Martin et al., 2017).

A TND é indicada para tratamentos que incluem: doenças relacionadas ao sistema gastrointestinal, as quais são causadas pela má absorção e digestão dos nutrientes, doenças do sistema neurológico, malformações, dentre outras. Para o início da terapia nutricional, portanto, se faz necessário que um profissional qualificado na área, entenda o diagnóstico de seu paciente e prescreva uma dieta individualizada que contemple os macro e micronutrientes necessários para os objetivos terapêuticos a fim de restabelecer a saúde do paciente (Zaban et al., 2010; Tinoco, 2013).

A nutrição domiciliar é considerada um tratamento econômico e seguro, visto que diminui os gastos com internação e evita as infecções hospitalares. Por outro lado, deve-se levar em consideração que apesar dos pontos positivos, as dietas domiciliares estão sucintas a contaminação microbiológica. Por essa razão, a fim de garantir a adequabilidade dessas, todas as etapas de higienização devem ser realizadas e respeitadas com muito critério e rigor (Lucas, Ribeiro, Furtado, \& de Menezes, 2018).

A Constituição da República Federativa do Brasil (CRFB), impõe que a alimentação é um direito garantido para todos, devendo estar em condições adequadas para o consumo, portanto, pode-se afirmar que a TND é apoiada por lei. Ademais, o programa "melhor em casa", auxilia ao acesso de dietas individualizadas à pacientes que vivem em condições precárias (Brasil, 2016; Buss et al., 2007). 
Assim sendo, a fim de compreender claramente como funciona a TND, e quais são seus benefícios e pontos negativos, o objetivo do presente trabalho foi a elaboração de uma revisão bibliográfica voltada aos aspectos da TND.

\section{Metodologia}

Este estudo refere-se a uma revisão narrativa qualitativa (Pereira et al., 2018), que tem por finalidade discutir, e ampliar o conhecimento sobre a nutricional oral/enteral/parenteral no âmbito domiciliar. Essa revisão é constituída por uma análise ampla da literatura, sabendo que se faz essencial a aquisição do conhecimento sobre o tema em foco, bem como a atualização do mesmo.

Utilizou-se para o levantamento dos principais manuscritos relacionados ao tema, plataformas como PubMed (US National Library of Medicine), Scientific Electronic Library Online Brasil (SciELO), Web of Science e Portal Periodicos Capes, tendo como período de referência os últimos 15 anos. O método utilizado para a inclusão das publicações foi conter no título, palavras-chaves e no resumo, termos vinculados a nutrição oral/enteral/parenteral, terapia nutricional domiciliar, assistência dos órgãos de saúde, e redução dos leitos hospitalares.

Seguidamente a escolha dos artigos, foi realizada, uma leitura atenciosa, destacando os principais tópicos e direcionando uma discussão acerca deste.

\section{Resultados e Discussão}

\subsection{Terapia Nutricional Domiciliar}

Para manter a manutenção da homeostase do organismo, a alimentação se torna um fator primordial e extremamente importante, especialmente para aqueles acometidos por algum tipo de enfermidade. Nesses casos, um plano de cuidado nutricional individualizado é necessário, dentre este, inclui-se a Terapia Nutricional Domiciliar (TND) (Cutchma et al., 2016).

A TND, como o próprio nome diz, pode ser definida como um método assistencial eficaz utilizado para a recuperação e manutenção do aporte nutricional de pacientes não saudáveis, que se encontram em suas próprias casas ou em instituições de repouso (Ribas et al., 2016).

Historicamente, na década de 1960, apareceram os primeiros serviços brasileiros de atenção domiciliar (AD), a fim de reduzir a taxa de internação e ocupação hospitalar. Com o tempo, esses serviços aumentaram de forma gradativa e foram oficialmente regulamentados pelo Sistema Único de Saúde (SUS), em 2002. Por conseguinte, em 2006, a fim de garantir maior adequabilidade de tal serviço, a Agência Nacional de Vigilância Sanitária (ANVISA) publicou um regulamento que tratava do funcionamento dos serviços domiciliares. Devido a grande repercussão, em 2011, criou-se o programa "Melhor em Casa", englobando a AD no SUS e objetivando a importância da terapia nutricional em domicílio (Brasil, 2013; Brasil, 2014; Oliveira et al., 2021).

A TND pode ser administrada de forma oral, enteral ou parenteral, cabendo a um profissional adequado prescrever qual é mais eficaz para determinado paciente (De Legge, Ireton-Jones, 2007; Oliveira et al., 2021).

\subsubsection{Terapia Nutricional Domiciliar Oral (TNDO)}

$\mathrm{Na}$ Terapia Nutricional Domiciliar Oral (TNDO), recomenda-se o uso de alimentos especialmente formulados e processados nos quais são introduzidas modificações na composição em macro e micronutrientes, visando atender as necessidades de pessoas com condições metabólicas e fisiológicas específicas (Coppini et al., 2009).

A terapia oral é indicada quando o paciente não consegue atingir suas necessidades nutricionais via alimentação convencional. No entanto, é imprescindível uma avaliação com profissionais especializados, que irão determinar o tipo e quantidade do suplemento nutricional a ser utilizado, levando em consideração aspectos como doenças, alterações 
laboratoriais, utilização de fármacos, função digestória, estado nutricional, ingestão alimentar, necessidades nutricionais e de nutrientes específicos e aceitação do suplemento nutricional oral (Mitne, 2006).

\subsubsection{Terapia Nutricional Domiciliar Enteral (TNDE)}

A Nutrição enteral, pode ser definida como um procedimento no qual o fornecimento de nutrientes, é realizado diretamente ao sistema gastrointestinal, para aqueles pacientes que possuem doenças em fase aguda ou crônica, e que apresentam dificuldade para deglutir (Bolognese et al., 2021; Perote et al., 2014; Garita et al., 2009; Waitzber et al., 2016; Kreymann et al., 2006). Ainda, a NE é altamente indicada para pacientes com dificuldades para alcançar pelo menos $70 \%$ de suas necessidades nutricionais via oral (Waitzberg, 2004; Castro et al., 2018).

De forma geral, a administração da alimentação enteral, é realizada via sonda nasogástrica (tubo inserido através da cavidade nasal até o estômago), nasoenteral (tubo introduzido através da cavidade nasal até o intestino delgado), gastrostomia (tubo intragástrico percutâneo) ou por meio da jejunostomia (tubo enteral percutâneo) (Pradelli et al., 2012).

Muitas vezes, confunde-se a NE com alimentação convencional, porém se faz importante compreender que em ambas, há diferenças plausíveis. Enquanto a alimentação convencional está voltada para os sentidos normais do ser humano, como mastigação, deglutição, digestão, olfato e paladar, as dietas entéricas são estabelecidas por profissionais que entendem exatamente as necessidades nutricionais dos pacientes, por meio de diagnósticos realizados, além disso, as fórmulas enterais muitas vezes, não variam em composição de nutrientes, pelo contrário, são fixas e adequadas para determinado tipo de enfermidade (Wanamaker et al., 2004).

A NE pode ser administrada a domicílio, essas dietas compõem o que chamamos de Terapia Nutricional Domiciliar Enteral (TNDE). Segundo Ribas et al., (2016), a TNED, como o próprio nome diz, se caracteriza por ser um método eficaz, que deve ser prescrita por profissionais capacitados, como o nutricionista. Tais profissionais, possuem a função de orientar o paciente nutricionalmente, auxiliar na educação alimentar e intervir dieteticamente em caso de problemas de saúde.

Durante toda a avaliação nutricional, são realizados diversos exames bioquímicos, coleta de dados socioeconômicos, histórico familiar, dentre outros, tudo isso se faz essencial para o fornecimento de diagnósticos que permitem estabelecer uma conduta nutricional mais adequada (Oliveira et al., 2021). Ademais, autores como Szlagatys-Sidorkiewicz et al. (2012) define a TNED como um conjunto de procedimentos terapêuticos que possuem a função de recuperar o estado nutricional dos pacientes que estão impossibilitados de alcançar suas necessidades via alimentação convencional e garantir a funcionalidade íntegra do organismo, tudo feito de forma domiciliar.

\subsubsection{Terapia Nutricional Domiciliar Parenteral (TNDP)}

A Portaria 272, de 09 de abril de 1998, define a nutrição parenteral como sendo:

“(...) Solução ou emulsão, composta basicamente de carboidratos, aminoácidos, lipídios, vitaminas e minerais, estéril e apirogênica acondicionada em recipiente de vidro ou plástico, destinada à administração intravenosa a pacientes desnutridos ou não, em regime hospitalar, ambulatorial ou domiciliar, visando a síntese ou á manutenção dos tecidos, órgãos e sistemas. Ela pode ser via central (administrada por uma veia de grande diâmetro, geralmente subclávia ou jugular interna, que chega diretamente ao coração), ou periférica (administrada através de uma veia menor, geralmente na mão ou antebraço)."

A TNDP é amplamente indicada para aqueles pacientes que estão incapacitados de satisfazer suas necessidades nutricionais via oral e enteral, ou seja, casos em que o sistema digestório está comprometido e por consequência a absorção dos nutrientes é incompleta ou impossível (Martin et al., 2017).

Um ponto importante a se considerar é que a TNDP não é livre de riscos, e por isso ainda gera certos receios, porém 
enfatiza-se que os atendimentos em casa são realizados por profissionais habilitados e competentes indicados para tal serviço (Kirby et al.,2017).

\subsection{Indicação da Terapia Nutricional Domiciliar}

A TND é indicada para tratamentos que incluem: doenças relacionadas ao sistema gastrointestinal, as quais são causadas pela má absorção e digestão dos nutrientes, doenças do sistema neurológico, malformações, câncer, e outras enfermidades em menor recorrência. Deve-se compreender que a TND apresenta indicação similar a indicação hospitalar, visto que a continuidade do tratamento iniciada no hospital, é feita agora em ambiente domiciliar (Zaban et al., 2010; Tinoco, 2013).

Com relação a TNDP, a mesma é indicada para aqueles pacientes em que a nutrição oral e enteral está temporariamente impossibilitada ou em casos de distúrbios de absorção. Os casos clínicos mais comuns são: síndrome do intestino curto, câncer, isquemia mesentérica, pancreatite grave necrotizante, fístula digestiva, obstrução mecânica do intestino delgado inoperável, enterite actínica, síndrome de má absorção, hiperemese gravídica, fibrose cística, pacientes em préoperatório com desnutrição moderada ou grave, doença de Crohn grave, dentre outras (Ciosak, Matsuba, Silva, Serpa, \& Poltronieri, 2011).

Já a TNDE é amplamente indicada para aqueles pacientes que possuem redução na ingestão oral, e dessa forma não conseguem suprir seu aporte calórico apenas com a alimentação normal. Dentre os principais casos clínicos de TNDE destacam-se: doença inflamatória intestinal, neuropatias, queimaduras, desnutrição, disfagia, paciente gravemente enfermo com múltiplas enfermidades, quimioterapia e radioterapia (Pedrón-Giner, Calderón, Martínez-Zazo, Villaroya, \& González, 2012).

\subsection{Segurança Alimentar e Nutricional}

As dietas orais/enterais/parenterais caseiras ofertadas em domicílios, são preparadas a base de alimentos in natura, fontes de carboidratos, proteínas, lipídios, vitaminas, dentre outros nutrientes essenciais (Bolognese et al., 2021; Mauricio, Gazola, \& Matioli, 2008). Porém, apesar da praticidade, essas dietas estão amplamente sujeitas à contaminação microbiológica quando manipuladas em domicílio, devido a higiene inadequada dos utensílios utilizados e dos manipuladores, temperatura que colabora para a proliferação de micro-organismos, dentre outros (Lucas et al., 2018). Por essa razão, a fim de garantir a adequabilidade dessas, todas as etapas de higienização devem ser realizadas e respeitadas com muito critério e rigor (Maniglia, Pagnani et al., 2015; Lucas et al., 2018).

Furlaneto-Maia et al., (2009) avaliou as condições microbiológicas de cinco dietas enterais artesanais, e como resultado obteve que em todas elas foi possível identificar a presença de coliformes totais e fecais. Além disso, encontrou-se na superfície dos objetos utilizados para o preparo, cerca de 88\% de contaminação por S. aureus, $100 \%$ de coliformes totais e $11 \%$ de coliformes decais, e por fim, as superfícies das mãos dos manipuladores também estavam contaminadas. Um outro estudo realizado por Felicio et al., (2012), evidenciou que após as análises físico-químicas de formulações enterais preparadas manualmente, houve perda considerável de nutrientes e contaminação microbiológica por parte dessas.

Portanto, a partir do explícito acima, deve-se ter a conscientização que as dietas domiciliares precisam ser preparadas com o máximo de cuidado possível para não haver contaminação e prejudicar ainda mais a saúde do paciente em tratamento.

\subsection{Contribuições do Cuidador}

Diante da discussão acerca do tema "terapia nutricional domiciliar", é importante abordar sobre o papel que o cuidador assume frente ao paciente em tratamento. O mesmo auxilia sobretudo nas atividades diárias, como a alimentação, higienização, locomoção, entre outras (Brasil, 2008). 
Para desempenhar sua função da melhor forma possível, o mesmo deve ser orientado por profissionais da saúde e todas as atividades e decisões são sempre discutidas com a família do paciente em tratamento. Com relação a TND, o cuidador pode ser extremamente essencial na adesão do paciente e, por consequência na melhoria significativa deste, assim, quanto mais envolvido o cuidador estiver com o paciente, melhor será a adequação a nova rotina fora do ambiente hospitalar (Brasil, 2008).

Como, na maioria das vezes, o paciente é totalmente dependente do cuidador, o mesmo fica também encarregado por diversas funções, como: escolha, compra, higienização e armazenamento dos alimentos para preparação da fórmula nutricional; preparo, conservação e oferta correta das dietas; higienização dos materiais e utensílios utilizados no preparo; seguir corretamente o fracionamento e os horários de administração da fórmula nutricional; posicionamento adequado do paciente e identificação de complicações decorrentes do processo dietético. Todos esses pontos são de fato importantes e indispensáveis para a melhora rápida do paciente em tratamento (Brasil, 2008).

\subsection{Custo das Fórmulas Nutricionais Industrializadas}

A nutrição domiciliar é considerada um tratamento econômico e seguro, visto que diminui os gastos com internação e impede a contaminação que ocorre no ambiente hospitalar.

Uma pesquisa realizada por Kutz et al. (2018), evidencia que as dietas preparadas manualmente apresentam custo 8 vezes menor quando comparado as dietas industrializadas. Os resultados obtidos mostram que a dieta caseira 1, representa $11,88 \%$ do custo médio total para seu equivalente industrializado ( $\mathrm{R} \$ 218,04)$ e a $\mathrm{D} 2$ representa $12,32 \%$ do custo médio total para seu equivalente industrializado $(\mathrm{R} \$ 245,51)$.

Abaixo, encontra-se tabela contendo os valores e as indicações das dietas disponíveis no mercado.

As informações contidas na tabela estão presentes nos encartes dos produtos fornecidos pelo fabricante. Os preços foram cotados em lojas de produtos de nutrição entre os meses de março e abril de 2020 na cidade de Maringá, Paraná, Brasil. 
Tabela 1. Preço das Dietas enterais/orais disponíveis no mercado brasileiro, bem como sua indicação.

\begin{tabular}{|c|c|c|c|c|c|}
\hline Dieta & $\begin{array}{l}\text { Quantidade } \\
\text { (ml ou } \\
\text { gramas) }\end{array}$ & Fórmula & Preço & Enteral / Oral & Indicação \\
\hline 1 & 1000 & Liquida & $\mathrm{R} \$ 30,00$ & Normocalórica & Risco nutricional ou desnutrição leve, anorexia, convalecência \\
\hline 2 & 1000 & Liquida & $\mathrm{R} \$ 29,00$ & Normocalórica & Risco nutricional ou desnutrição leve, TNE por tempo prolongado e regularização do trânsito intestinal \\
\hline 3 & 1000 & Liquida & $\mathrm{R} \$ 39,00$ & Hiperproteica & Desnutrição moderada a grave com necessidade calórica elevada \\
\hline 4 & 1000 & Liquida & $\mathrm{R} \$ 39,00$ & Hiperproteica & $\begin{array}{c}\text { Desnutrição moderada a grave com necessidade calórica elevada, associada ou não à restrição hídrica, TNE prolongada e } \\
\text { regularização do trânsito intestinal }\end{array}$ \\
\hline 5 & 1000 & Liquida & $\mathrm{R} \$ 41,00$ & Hiperproteica & $\begin{array}{c}\text { Desnutriçãoproteico-calórica com necessidade elevada de proteína: pacientes graves de UTI (Sepse, Trauma, Queimados e } \\
\text { Cirúrgicos) }\end{array}$ \\
\hline 6 & 500 & Liquida & $\mathrm{R} \$ 40,00$ & Hiperproteica & $\begin{array}{l}\text { Pacientes graves de UTI associado à restrição hídrica severa e necessidade elevada de proteína (Trauma, Sépse, ICC, Obeso } \\
\text { crítico, DPOC, Pós operatório, IRA) }\end{array}$ \\
\hline 7 & 500 & Liquida & $\mathrm{R} \$ 35,00$ & Hiperproteica & $\begin{array}{c}\text { Pacientes graves com necessidades elevadas de proteína associada à restrição hídrica severa (ex: DPOC, Cardiopátas, } \\
\text { obesidade) e regularização do trânsito intestinal }\end{array}$ \\
\hline 8 & 1000 & Liquida & $\mathrm{R} \$ 71,00$ & Normocalórica & Distúrbios disabsortivos (pancreatite, Síndrome do intestino curto, Doença de Crohn, Síndrome de má absorção e Fístulas) \\
\hline 9 & 500 & Liquida & $\mathrm{R} \$ 39,00$ & Hiperproteica & Insuficiencia Hepática com risco ou presença de encefalopatia (cirrose hepática, pré-transplante de fígado) \\
\hline 10 & 500 & Liquida & $\mathrm{R} \$ 39,00$ & Hiperproteica & Atende as necessidades nutricionais dos pacientes com câncer em processo de caquexia \\
\hline 11 & 500 & Liquida & $\mathrm{R} \$ 45,00$ & Normocalórica & Indicado para crianças em risco nutricional ou desnutrição leve \\
\hline 12 & 500 & Liquida & $\mathrm{R} \$ 44,00$ & Normocalórica & Indicada para crianças em risco nutricional ou desnutrição leve. TNE prolongada, regularização do trânsito intestinal \\
\hline 13 & 500 & Liquida & $\mathrm{R} \$ 46,00$ & Hiperproteica & Indicada para crianças com desnutriçãomoderna a grave com necessidade calórica elevada, associada à restrição hídrica \\
\hline 14 & 500 & Liquida & $\mathrm{R} \$ 41,00$ & Hiperproteica & $\begin{array}{l}\text { Indicada para crianças com desnutrição moderada a grave com necessidade calórica elevada, associada à restrição hídrica e } \\
\text { necessidade de regularização do trânsito intestinal }\end{array}$ \\
\hline 14 & 400 & Pó & $\mathrm{R} \$ 100,00$ & Normocalórica & Para pacientes críticos com intolerância GI e/ou dificuldadena absorção da proteína intacta \\
\hline 16 & 1000 & Liquido & $\mathrm{R} \$ 162,00$ & Normocalórico & Para pacientes críticos com intolerância GI e/ou dificuldade na absorção da proteína intacta \\
\hline
\end{tabular}


Research, Society and Development, v. 11, n. 3, e34011326130, 2022

(CC BY 4.0) | ISSN 2525-3409 | DOI: http://dx.doi.org/10.33448/rsd-v11i3.26130

\begin{tabular}{|c|c|c|c|c|c|}
\hline 17 & 1000 & Liquido & $\mathrm{R} \$ 30,00$ & hipercalorica & $\begin{array}{l}\text { Para pacientes críticos com intolerância GI e/ou com dificuldade na absorção da proteína intecta, com alta necessidade } \\
\text { calórica }\end{array}$ \\
\hline 18 & 500 & Liquido & $\mathrm{R} \$ 79,00$ & $\begin{array}{l}\text { Hipercalorica e } \\
\text { hiperproteica }\end{array}$ & $\begin{array}{c}\text { Pacientes críticos com intolerância gastrointestinal e/ou dificuldade na absorção da proteína intácta, que necessitam iniciar a } \\
\text { nutrição em menor volume }\end{array}$ \\
\hline 19 & 1000 & Liquido & $\mathrm{R} \$ 146,00$ & normocalórico & $\begin{array}{l}\text { Pacientes críticos com intolerância GI e/ou com dificuldade na absorção da proteína intacta, que necessitam de altas } \\
\text { quantidades de proteínas e menos CHO }\end{array}$ \\
\hline 20 & 1000 & Liquido & $\mathrm{R} \$ 122,00$ & $\begin{array}{l}\text { Hipercalorica e } \\
\text { hiperproteica }\end{array}$ & Pacientes que necessitam de nutrição com maior aporte calórico e proteico \\
\hline 21 & 1000 & Liquido & $\mathrm{R} \$ 12,00$ & hipercalorico & $\begin{array}{l}\begin{array}{l}\text { Paceintes renais agudos ou crônicos em tratamento dialítico que necessitem de maior aporte calórico-proteico e restrição de } \\
\text { volume }\end{array} \\
\text { valu }\end{array}$ \\
\hline 22 & 1000 & Liquido & $\mathrm{R} \$ 18,00$ & Normocalórica & $\begin{array}{l}\text { Preparo imunológico pré e pós-operatório de cirurgia de grande porte (cirurgias oncológicas do TGI, cirurgia de cabeça e } \\
\text { pescoço, entre outras) }\end{array}$ \\
\hline 23 & 1000 & Liquido & $\mathrm{R} \$ 220,00$ & Hiperproteica & $\begin{array}{l}\text { Preparo imunológico pré e pós-operatório de cirurgia de grande porte (cirurgias oncológicas do TGI, cirurgia de cabeça e } \\
\text { pescoço, entre outras) }\end{array}$ \\
\hline 24 & 200 & Liquido & $\mathrm{R} \$ 16,50$ & hiperproteico & Pacientes com problema de cicatrização no geral, como lesões por pressão, lesões crônicas, pé diabético etc. \\
\hline 25 & 1000 & Liquido & $\mathrm{R} \$ 59,00$ & hipercalorico & Pacientes com diarreia aguda ou crônica que necessitam de fibras solúveis e maior aporte calórico \\
\hline 26 & 1000 & Liquido & $\mathrm{R} \$ 17,50$ & normocalorica & Manutenção ou recuperação do estado nutricional. Opção para paciente domiciliar \\
\hline 27 & 1000 & Liquido & $\mathrm{R} \$ 19,00$ & normocalorica & Manutenção ou recuperação do estado nutricional de pacientes que necessitam de fibras. Opção para paciente domiciliar \\
\hline 28 & 1000 & Liquido & $\mathrm{R} \$ 23,00$ & normocalorica & Manutenção ou recuperação do estado nutricional de pacientes que necessitam da adição de um mix de fibras \\
\hline 29 & 1000 & Liquido & $\mathrm{R} \$ 25,00$ & Hiperproteica & Manutenção ou recuperação do estado nutricional de pacientes com maiores necessidades calóricas e/ou restrição de volume \\
\hline 30 & 1000 & Liquido & $\mathrm{R} \$ 54,00$ & Hiperproteica & $\begin{array}{c}\text { Manutenção ou recuperação do estado nutricional de pacientes com maiores necessidades calóricas e/ou com restrição de } \\
\text { volume e de fibras }\end{array}$ \\
\hline 31 & 1000 & Liquido & $\mathrm{R} \$ 26,00$ & hiperproteico & Manutenção ou recuperação do estado nutricional de pacientes que necessitam de um maior aporte proteico \\
\hline 3 & 400 & Pó & $\mathrm{R} \$ 45,00$ & Normocalórica & Manutenção ou recuperação do estado nutricional \\
\hline 33 & 200 & Liquido & 8,00 & Hiperproteica & $\begin{array}{l}\begin{array}{l}\text { Manutenção ou recuperação do estado nutricional de pacientes que necessitam de altas doses calóricas e/ou com restrição de } \\
\text { volume }\end{array}\end{array}$ \\
\hline 34 & 200 & Liquido & $\mathrm{R} \$ \quad 12,00$ & Hiperproteica & $\begin{array}{c}\text { Manutenção ou recuperação do estado nutricional de pacientes que necessitam de altas doses calóricas e/ou com restrição de } \\
\text { volume }\end{array}$ \\
\hline
\end{tabular}


Research, Society and Development, v. 11, n. 3, e34011326130, 2022

(CC BY 4.0) | ISSN 2525-3409 | DOI: http://dx.doi.org/10.33448/rsd-v11i3.26130

\begin{tabular}{|c|c|c|c|c|c|}
\hline 35 & 200 & Liquido & $\mathrm{R} \$ 10,00$ & normocalorica & Pacientes com necessidade de controle glicêmico \\
\hline 36 & 1000 & Liquido & $\mathrm{R} \$ 86,00$ & Hiperproteica & Pacientes com necessidade de controle glicêmico que necessitam de maior aporte calórico e proteico \\
\hline 37 & 1000 & Liquido & $\mathrm{R} \$ 78,00$ & hiperproteica & Necessidade de controle glicêmico que precisam de maior aporte proteico \\
\hline 38 & 400 & Pó & $\mathrm{R} \$ 48,00$ & normocalorica & $\begin{array}{l}\text { Para crianças de } 1 \text { a } 10 \text { anos de idade, sem problemas de absorção, que necessitam de nutrição adequada para recuperação e } \\
\text { manutençào do estado nutricional }\end{array}$ \\
\hline 39 & 250 & Liquido & $\mathrm{R} \$ 30,00$ & normocalorica & $\begin{array}{c}\text { Para pacientes pediátricos críticos de } 4 \text { a } 10 \text { anos de idade, com intolerância GI e/ou dificuldade da absorção da proteína } \\
\text { intácta }\end{array}$ \\
\hline 40 & 400 & Pó & $\mathrm{R} \$ 140,00$ & normocalorica & $\begin{array}{l}\text { Para pacientes pediátricos críticos de } 4 \text { a } 10 \text { anos de idade, com intolerância GI e/ou dificuldade da absorção da proteína } \\
\text { intácta }\end{array}$ \\
\hline 41 & 400 & Pó & $\mathrm{R} \$ 95,00$ & normocalorica & $\begin{array}{l}\text { Lactentes e crianças na primeira infância, de } 0 \text { a } 36 \text { m. com alergia às proteínas intáctas do leite de vaca e soja, sem } \\
\text { comprometimento do TGI e/ou sem restrição à lactose }\end{array}$ \\
\hline 42 & 400 & Pó & $\mathrm{R} \$ 128,00$ & normocalorica & $\begin{array}{l}\text { Lactentes e crianças na primeira infância, de } 0 \text { a } 36 \mathrm{~m} \text {. com alergia às proteínas intáctas do leite de vaca e soja, com } \\
\text { comprometimento do TGI e/ou com restrição à lactose }\end{array}$ \\
\hline 43 & 400 & Pó & $\mathrm{R} \$ 160,00$ & normocalorica & $\begin{array}{l}\text { Lactentes e crianças na primeira infância, de } 0 \text { a } 36 \text { m. com alergia às proteínas intáctas do leite de vaca e soja, com } \\
\text { comprometimento do TGI e/ou com restrição à lactose }\end{array}$ \\
\hline 44 & 800 & Pó & $\mathrm{R} \$ 76,00$ & normocalorica & $\begin{array}{c}\text { Desnutrição, anorexia nervosa, neoplasias, cardiopatias, doença neurológica e com necessidade de regularização do transito } \\
\text { intestinal }\end{array}$ \\
\hline 45 & 125 & Liquido & $\mathrm{R} \$ 39,00$ & Normocalórica & $\begin{array}{l}\text { Cardiopatia congenia, fibrose cística, insuficiencia respiratória, déficit pondero-estatural ou desaceleração do crescimento, } \\
\text { pré e pós operatório, restrição hidrica, intolerância }\end{array}$ \\
\hline 46 & 400 & Pó & $\mathrm{R} \$ 149,00$ & Normocalórica & $\begin{array}{l}\text { Cardiopatia congenia, fibrose cística, insuficiencia respiratória, déficit pondero-estatural ou desaceleração do crescimento, } \\
\text { pré e pós operatório, restrição hidrica, intolerância }\end{array}$ \\
\hline 47 & 500 & Liquido & $\mathrm{R} \$ \quad 26,90$ & Hiperproteica & Pacientes críticos, queimados, desnutridos com depleção proteica, pré e pós cirúrgicos \\
\hline 48 & 500 & Liquido & $\mathrm{R} \$ 45,00$ & Hiperproteica & Pacientes críticos, queimados, desnutridos com depleção proteica, pré e pós cirúrgicos \\
\hline 49 & 1000 & Liquido & $\mathrm{R} \$ 39,00$ & Normocalórica & $\begin{array}{c}\text { Paciente em risco nutricional, desnutrição,anorexia, pré e pós cirurgia, UTI, neoplasias, doenças neurológicas, cardiopatas, } \\
\text { realimentação }\end{array}$ \\
\hline 50 & 1000 & Liquido & $\mathrm{R} \$ 21,00$ & Hiperproteica & Risco nutricional ou situações de nutrição enteral prolongada \\
\hline 51 & 1000 & Liquido & $\mathrm{R} \$ 26,00$ & hiperproteica & Necessidade elevada de calorias associada ou não à restrição hídrica \\
\hline 52 & 1000 & Liquido & $\mathrm{R} \$ 26,00$ & Hiperproteica & Necessidades elevadas de proteínas, ideal para pacientes com caquexia ou câncer \\
\hline
\end{tabular}


Research, Society and Development, v. 11, n. 3, e34011326130, 2022

(CC BY 4.0) | ISSN 2525-3409 | DOI: http://dx.doi.org/10.33448/rsd-v11i3.26130

\begin{tabular}{|c|c|c|c|c|c|}
\hline 53 & 1000 & Liquido & $\mathrm{R} \$ 24,00$ & Hiperproteica & Nessecidade de regularização do trânsito intestinal e situações de nutrição enteral prolongada \\
\hline 54 & 1000 & Liquido & $\mathrm{R} \$ 17,00$ & Hiperproteica & Risco nutricional ou situações de nutrição enteral prolongada \\
\hline 55 & 380 & Pó & $\mathrm{R} \$ 40,00$ & Hiperproteica & Criança com risco nutricional ou desnutridas, que necessitam de nutrição enteral prolongada \\
\hline 56 & 200 & Liquido & $\mathrm{R} \$ 10,00$ & Normocalórica & Controle glicemico e prevenção das complicações do diabetes \\
\hline 57 & 400 & Pó & $\mathrm{R} \$ 87,00$ & Normocalórica & Distúrbios e sintomas digestivos e absortivos \\
\hline 58 & 200 & Liquido & $\mathrm{R} \$ 13,00$ & Hiperproteica & Recuperação nutricional de pacientes em processo dialítico \\
\hline 59 & 200 & Liquido & $\mathrm{R} \$ 52,00$ & Normocalórica & Ideal para pacientes oncolígicos, com alterações no apetite e paladar e com necessidade elevada de proteína \\
\hline 60 & 200 & Liquido & $\mathrm{R} \$ \quad 9,00$ & Hiperproteica & Risco nutricional, caquexia, AIDS e lesão por pressão \\
\hline 61 & 400 & Pó & $\mathrm{R} \$ \quad 46,00$ & Normocalórica & $\begin{array}{c}\text { Adequado para necessidades indivíduais e/ou condições clínicas específicas para recuperação e/ou manutenção do estado } \\
\text { nutricional }\end{array}$ \\
\hline 62 & 200 & Liquido & 9,00 & Hiperproteica & $\begin{array}{l}\text { Alimento adequado para manutenção ou recuperação do estado nutricional de pacientes que necessitem de um adequado } \\
\text { aporte calórico }\end{array}$ \\
\hline 63 & 200 & Liquido & $\mathrm{R} \$ 21,00$ & Hiperproteica & $\begin{array}{l}\text { Recuperação do estado nutricional e/ou sarcopenia. Adequado para recuperação da massa muscular, para adultos e idosos } \\
\text { em situação de fragilidade }\end{array}$ \\
\hline 64 & 220 & Liquido & $\mathrm{R} \$ 13,00$ & Hiperproteica & 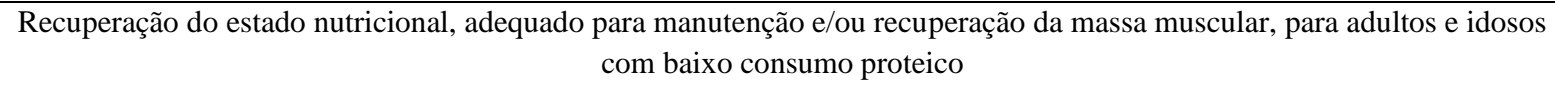 \\
\hline 65 & 1000 & Liquido & $\mathrm{R} \$ \quad 42,00$ & Normocalórica & $\begin{array}{l}\text { Alimento indicado para pacientes com necessidades de controle glicêmico, situação de estresse metabólico e/ou pacientes } \\
\text { diabéticos tipo I ou II }\end{array}$ \\
\hline 66 & 200 & Liquido & $\mathrm{R} \$ \quad 14,00$ & Hiperproteica & $\begin{array}{c}\text { Pacientes com necessidades de controle glicêmico, situação de estresse metabólico, pacientes diabeticos tipo I e II, maior } \\
\text { aporte calórico e proteico e restrição de volume }\end{array}$ \\
\hline 67 & 250 & Liquido & 7,00 & Hiperproteica & $\begin{array}{l}\text { Pacientes com necessidade de regulação da função intestinal, tratando tanto quadros de diarréia quanto constipação, com } \\
\text { adequado aporte calórico e proteico }\end{array}$ \\
\hline 68 & 237 & Liquido & $\mathrm{R} \$ 10,00$ & Hiperproteica & $\begin{array}{c}\text { Pacientes com necessidade de regulação da função intestinal, tratando tanto quadros de diarréia quanto constipação, com } \\
\text { adequado aporte calórico e proteico }\end{array}$ \\
\hline 69 & 400 & Pó & $\mathrm{R} \$ 46,00$ & Normocalórica & $\begin{array}{c}\text { Indicado para criança acima de } 1 \text { ano de idade, com necessidade de um melhor aporte calórico e proteico para recuperação } \\
\text { do estado nutricional e regulação do TGI }\end{array}$ \\
\hline
\end{tabular}

Fonte: Autores. 
Observa-se que dentre as dietas orais/enterais analisadas, a de número 67 (hiperproteica), é a que apresentou menor preço, 7 reais. Essa é indicada para pacientes com necessidade de regulação da função intestinal, tratando tanto quadros de diarreia quanto constipação, com adequado aporte calórico e proteico. Já a dieta número 23 , também definida como hiperproteica, apresentou maior preço, cerca de 220 reais. Esta é indicada para o preparo imunológico pré e pós-operatório de cirurgia de grande porte (cirurgias oncológicas do TGI, cirurgia de cabeça e pescoço, entre outras).

\subsection{Assistência dos Órgãos de Saúde}

As fórmulas de nutrição oral/enteral/parenteral são entregues por agentes comunitários aos pacientes em estado de vulnerabilidade em seus domicílios, com a presença de cuidadores. Sabe-se que muitas famílias vivem em estado de vulnerabilidade econômica, higiênico-sanitária e social, assim o acesso integral as dietas industrializadas e aos utensílios e alimentos adequados para o preparo das dietas caseiras, acaba sendo restrito (Mezzomo et al., 2021).

A Constituição da República Federativa do Brasil (CRFB) impõe que a alimentação é um direito garantido para todos, devendo estar em condições adequadas para o consumo (Brasil, 2016; Buss et al., 2007). Assim sendo, a TND também se torna um direito para aqueles que precisam (Rubia et al., 2020).

No Brasil, tem-se um programa denominado "Melhor em Casa", o mesmo atende usuários do SUS e fornece condições apropriadas para realizar o cuidado e atenção nutricional ao paciente. Isso é de fato importante, pois garante segurança as famílias mais carentes e diminuição dos gastos hospitalares (Rubia et al., 2020; Brasil, 2013).

\subsection{Melhoria na Qualidade de vida dos Pacientes}

As dietas domiciliares quando bem manipuladas e aplicadas, geram inúmeros benefícios para os pacientes, especialmente na redução da desnutrição e por consequência nas taxas de mortalidade. Ademais, a diminuição nos riscos de complicações severas, menor tempo de internação, rotatividade dos leitos, redução das despesas derivadas da hospitalização, diminuição de infecções hospitalares, são pontos importantes que reforçam a implementação e importância da TND.

Outro ponto importante a ser destacado, é que na TND, o paciente tem a oportunidade de recuperar-se em seu ambiente familiar, sentindo-se acolhido e próximo dos seus entes queridos. Sem dúvidas, é um ganho incontestável, diminuindo o tempo de reabilitação do estado nutricional, capacidade funcional e mental (Day, 2017).

\section{Considerações Finais}

A partir do estudo realizado, pode-se concluir que a TND é importante para a recuperação do estado nutricional do paciente debilitado. No entanto, para dar início ao tratamento é necessário que um profissional qualificado faça seu diagnóstico e prescreva a melhor fonte de nutrientes que o paciente necessita. Ademais, ressalta-se que a terapia nutricional pode ser dividida em oral, enteral e parenteral e que a melhor escolha será por meio de uma avaliação individualizada.

Apesar dos fatores positivos associados a esta, deve-se lembrar que a TND apresenta o risco de contaminação microbiológica, e por isso deve ser preparada com toda a higiene e rigor possível. Desta forma, recomenda-se maiores estudos nessa temática, que analisem o risco de contaminação na terapia nutricional enteral domiciliar em virtude do contato prolongado do manipulador com o alimento e a falta de local específico para manipulação da dieta.

Por fim, salienta-se que todos devem ter acesso a alimentação e total reintegração de sua saúde.

\section{Agradecimentos}

Os autores agradecem o Conselho Nacional de Desenvolvimento Científico e Tecnológico (CNPq) e a Coordenação de Aperfeiçoamento de Pessoal de Nível Superior (CAPES) pelo auxílio financeiro. 


\section{Referências}

Bolognese, M. A., de Souza, P. M., Néia, V. J. C., Santos, O. O., \& Visentainer, J. V. (2021). Nutrição enteral com ênfase na composição lipídica: uma revisão. Research, Society and Development, 10(15), e506101523178-e506101523178.

Brasil. (2008). Ministério da Saúde. Secretaria de Atenção à Saúde. Secretaria de Gestão do Trabalho e da Educação na Saúde. Guia prático do cuidador / Ministério da Saúde, Secretaria de Atenção à Saúde, Secretaria de Gestão do Trabalho e da Educação na Saúde. - Brasília : Ministério da Saúde. https://bvsms.saude.gov.br/bvs/publicacoes/guia_pratico_cuidador.pdf

Brasil. (2013). Ministério da Saúde. Secretaria de Atenção à Saúde. Departamento de Atenção Básica. Caderno de atenção domiciliar / Ministério da Saúde, Secretaria de Atenção à Saúde, Departamento de Atenção Básica. - Brasília : Ministério da Saúde. http://189.28.128.100/dab/docs/portaldab/publicacoes/cad_vol2.pdf

Brasil. (2014). Ministério da Saúde. Atenção domiciliar no SUS: Resultados do laboratório de inovação em atenção domiciliar / Ministério da Saúde. Organização $\quad$ Pan-Americana $\quad$ da $\quad$ Saúde. $\quad$ Saúde. https://bvsms.saude.gov.br/bvs/publicacoes/atencao_domiciliar_sus_resultados_laboratorio_inovacao.pdf

Brasil. (2016). Constituição da República Federativa do Brasil de 1988: texto constitucional promulgado em 5 de outubro de 1988, com as alterações determinadas pelas Emendas Constitucionais de Revisão nos 1 a 6/94, pelas Emendas Constitucionais nos 1/92 a 91/2016 e pelo Decreto Legislativo no 186/2008. - $\quad$ Brasília: $\quad$ Senado $\quad$ Federal, Coordenação $\quad$ de $\quad$ Edições https://www2.senado.leg.br/bdsf/bitstream/handle/id/518231/CF88_Livro_EC91_2016.pdf

Buss, P. M., \& Pellegrini Filho, A. (2007). A saúde e seus determinantes sociais. Physis: revista de saúde coletiva, 17, 77-93.

Castro e Cardoso, M. G., Prates, S. M. S., \& Anastácia, L. R. (2018). Fórmulas para nutrição enteral padrão e modificada disponíveis no Brasil: Levantamento e classificação. BRASPEN Journal, 33, 402-417. http://arquivos.braspen.org/journal/out-dez-2018/08formulas.pdf

Ciosak, S. I., Matsuba, C. S. T., Silva, M. L. T., Serpa, L. F., \& Poltronieri, M. J. (2011). Acessos para terapia de nutrição parenteral e enteral. Sociedade Brasileira de Nutrição Parenteral e Enteral e Associação Brasileira de Nutrologia [internet], 2-10.

Coppini, L. Z. C.; Waitzberg, D. L. (2009). Complicações em nutrição enteral. In: Waitzberg, D. L. (2009). Nutrição oral, enteral e parenteral na prática clínica. 4. ed. v. 2. São Paulo: Atheneu, 907-917.

Cutchma, G., Mazur, C. E., Thieme, R. D., De Franca, R. M., \& Schieferdecker, M. M. (2016). Nutrition formulas: influence on nutritional condition, clinical condition and complications in household nutrition therapy. Nutrición Clínica y Dietética Hospitalaria, 36(2), 45-54.

Day, T. (2017). Home enteral feeding and its impact on quality of life. British journal of community nursing, 22(Sup7), S14-S16.

De Legge, M. H., \& Ireton-Jones, C. (2007). Home care. In: Gottschlich, M. M.; De Legge, M. H,; Mattox, T.; Mueller, C.; Worthington, P. The ASPEN nutrition support core curriculum: a case-based approach - the adult patient. Silver Spring: American Society for Parenteral and Enteral Nutrition.

Felicio, B. A., Pinto, R. O. M., Andrade, N., \& Da Silva, D. F. (2012). Food and nutritional safety of hospitalized patients under treatment with enteral nutrition therapy in the Jequitinhonha Valley, Brazil. Nutricion hospitalaria, 27(6), 2122-2129.

Furlaneto-Maia, L., \& Pangoni, G. (2009). Avaliação microbiológica de preparações artesanais de dietas enteral em uma Unidade de Alimentação e Nutrição. Journal of Health Sciences, 11(1).

Garita, F. S., Cukier, C., \& Magnoni, D. (2009). Indicações e prescrição da terapia nutricional. Matusuba CST, Magnoni D, organizadores. Enfermagem em terapia nutricional. São Paulo: SARVIER, 35-55.

Kirby, D. F., Corrigan, M. L., Hendrickson, E., \& Emery, D. M. (2017). Overview of home parenteral nutrition: an update. Nutrition in Clinical Practice, 32(6), 739-752.

Kreymann, K. G., Berger, M. M., Deutz, N. E., Hiesmayr, M., Jolliet, P., Kazandjiev, G., ... \& Spies, C. E. S. P. E. N. (2006). ESPEN guidelines on enteral nutrition: intensive care. Clinical nutrition, 25(2), 210-223.

Kutz, N. A., de Souza Bonfim, V. A., Assis, A. L., Barbosa, M. C., \& da Silva, N. M. (2018). Padronização de dietas enterais artesanais para uso domiciliar na Atenção Primária. Revista Família, Ciclos de Vida e Saúde no Contexto Social, 1, 298-305.

Lucas, J. L. L., Ribeiro, J. C., Furtado, C. C., \& de Menezes, P. M. G. (2018). Comparação entre Dietas Enterais Artesanais e Industrializadas: Uma Revisão da Literatura. UNILUS Ensino e Pesquisa, 15(38), 5-10.

Maniglia, F. P., Pagnani, A. C. C., \& Nascimento, G. G. (2015). Desenvolvimento de dieta enteral artesanal com propriedades funcionais. Rev. Bras. Nutr. Clin, 30(1), 66-70.

Martin, K., \& Gardner, G. (2017). Home enteral nutrition: updates, trends, and challenges. Nutrition in Clinical Practice, 32(6), 712-721.

Mauricio, A. A., Gazola, S., \& Matioli, G. (2008). Non industrialized enteral diets: microbiological analysis and verification of good preparation practices. Revista de Nutricao-Brazilian Journal of Nutrition, 21(1), 29-37.

Mezzomo, R. M., Fiori, S. L. S., Reis, O. L. O., \& Schieferdecker, M. E. M. (2021). Nutritional composition and cost of home-prepared enteral tube feeding. Clinical Nutrition ESPEN, 42, 393-399.

Mitne, C. (2006). Preparações não-industrializadas para nutrição enteral. In: Waitzberg, D. L. Nutrição oral, enteral e parenteral na prática clínica. 3. ed. São Paulo: Atheneu, 629-657. 
Research, Society and Development, v. 11, n. 3, e34011326130, 2022

(CC BY 4.0) | ISSN 2525-3409 | DOI: http://dx.doi.org/10.33448/rsd-v11i3.26130

Oliveira, J. P. L. D., Silva, C. F. X. C. D. A., Ferreira, L. G., \& Teixeira, L. G. (2021). Aspectos clínicos de pacientes infanto-juvenis em terapia nutricional enteral domiciliar: uma revisão integrativa. Physis: Revista de Saúde Coletiva, 31 (2), e310216.

Pedrón-Giner, C., Calderón, C., Martínez-Zazo, A., Villaroya, E. C., \& González, P. M. (2012). Home enteral nutrition in children; a 10 year experience with 304 pediatric patients. Nutricion hospitalaria, 27(5), 1444-1450.

Perote, G. M., Vieira, R. Q, \& Medeiros, J. L. (2014). Nutrição enteral e risco de contaminação microbiológica: uma revisão de literatura. Nutrivisa - Revista de Nutrição e Vigilância em Saúde, 1, 23-26. https://www.revistanutrivisa.com.br/wp-content/uploads/2014/11/nutrivisa-vol-1-num-3-e.pdf.

Pradelli, L., Mayer, K., Muscaritoli, M., \& Heller, A. R. (2012). n-3 fatty acid-enriched parenteral nutrition regimens in elective surgical and ICU patients: a meta-analysis. Critical Care, 16(5), 1-10.

Ribas, D. F., Buzzini, R. F., Simões, R. S., Kelman, G., \& Bernardo, W. M. (2016). Nutrition assessment-Home-based nutritional therapy. Revista da Associação Médica Brasileira, 62, 711-717.

Rubia, T. D., Schieferdecker, M. E. M., \& Ditterich, R. G. (2020). Older adults undergoing home enteral nutrition therapy: integration of national public policy and municipal programs. Revista Brasileira de Geriatria e Gerontologia, 23(6), 200157.

Szlagatys-Sidorkiewicz, A., Popińska, K., Toporowska-Kowalska, E., Borkowska, A., Sibilska, M., Gębora-Kowalska, B., ... \& Książyk, J. (2012). Home enteral nutrition in children-2010 nationwide survey of the polish society for clinical nutrition of children. European journal of pediatrics, 171 (4), 719-723.

Tinoco, A. C., Pino, P., Zavala, A., \& Hodgson, M. I. (2013). Evaluación clínica del uso de nutrición enteral domiciliaria en pacientes pediátricos. Revista chilena de nutrición, 40(1), 21-25.

Waitzber, D. L., \& Torrinhas, R. S. (2016). Enteral Feeding. Reference Module in Food Science Encyclopedia of Food and Health.

Waitzberg, D. L. (2004). Nutrição oral, enteral e parenteral na prática clínica. In Nutrição oral, enteral e parenteral na prática clínica, 1858 -1858.

Wanamaker, R., \& Grimm, I. (2004). Encyclopedia of Gastroenterology. Gastroenterology, 127(4), 1274-1275.

Zaban, A. L. R. S., \& Novaes, M. R. C. G. (2010). Home enteral nutrition in children: a one-year experience with 184 patients in Distrito Federal, Brazil Experimental Biology and Medicine, 235 (1), 584-589. 